\title{
LES KYSTES A DIDYMOCYSTIS WEDLI DU THON ÉTUDE ANATOMO-PATHOLOGIQUE
}

\author{
Par Jean TIMON-DAVID
}

Le thon rouge (Thynnus thynnus L.) est très fréquemment parasité en Méditerranée par différents types de Trématodes appartenant à la remarquable famille des Didymozoonidæ. J'ai signalé, en 1935 , les Wedlia de l'estomac et les lésions qu'ils provoquent.

L'espèce de ce groupe la plus commune est certainement Didymocystis wedli Ariola : je l'ai souvent observée sur les branchies des thons de la côte provençale; son anatomie est bien connue depuis les récents travaux de Yamaguti (1934), qui a révisé les anciennes descriptions d'Ariola et établi la synonymie des formes décrites ultérieurement (Didymocystis kobayashii R.-Ph. Dollfus, 1926 = Wedlia katsuwonicola Okada, 1926) ; mais on ignore tout de sa biologie. C'est pour tenter d'apporter quelques précisions sur le mode de vie de ce parasite que j'ai réalisé le présent travail, dans lequel je me propose d'étudier les caractères anatomo-pathologiques des branchies de thon envahies par les Didymocystis.

Matériel et techniques. - Le matériel qui a servi à ces recherches provient du port de Carro (B.-du-R.), où il a été recueilli, en février 1937, par les pêcheurs de cette localité, spécialisés dans la capture hivernale des thons.

Les feuillets branchiaux ont été fixés au Bouin quelques heures après la mort des poissons; les coupes ont été orientées suivant différentes directions, mais les plus démonstratives ont été obtenues suivant le plan perpendiculaire au feuillet brachial (voir pl. XV, les microphotographies). Les préparations ont été colorées à l'hémalun-éosine, aux trichromiques de Cajal (magenta-picro-carmin d'indigo), et de Masson (hémalun-érythrosine-safran); les clichés ont été obtenus sur plaques panchromatiques.

Aspect macroscopique. - A l'examen direct, les kystes à Didymocystis se présentent comme des nodosités elliptiques siégeant sur le bord libre du feuillet branchial ; cette situation est rigoureusement constante (fig.); leur couleur jaune-doré tranche sur la

Annales de Parasitologie, $\mathrm{T}, \mathrm{XV}, \mathrm{N}^{\circ} 6,-1^{\mathrm{er}}$ novembre 1937 , p. 520-523. 
teinte rougeâtre de la branchie et permet de les repérer facilement. Les dimensions des kystes sont les suivantes: grand axe, parallèle au bord du feuillet, 2,5 à $4 \mathrm{~mm}$., petit axe 0,9 à $1 \mathrm{~mm}$., 2 .

Il est facile de dissocier la paroi au moyen de deux aiguilles sous le contrôle du binoculaire : on peut ainsi extraire les parasites qui sont toujours groupés deux à deux.

Pour bien comprendre la localisation des parasites et pour suivre la formation des kystes, il est indispensable d'étudier, au préalable, la structure histologique du feuillet branchial normal.

Le feuillet branchial du thon. - Le feuillet branchial est constitué à l'état normal par un

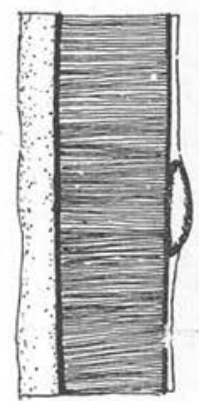

Fig. - Aspect d'un fragment de feuillet branchial de thon, portant un kyste à Didymocystis (grossi deux fois). axe ostéo-cartilagineux (Kiemengräte, arête branchiale d'Oppel), épaissi en triangle du côté proximal et atténué du côté distal. Cet axe est large seulement de $10 \mu$ (en moyenne) dans sa partie médiane.

Les deux bords du feuillet sont parcourus par les vaisseaux afférent et efférent qui occupent toujours une situation marginale : la veine sur le bord externe, l'artère sur le bord interne. La veine est protégée par une gouttière osseuse, en relations avec l'axe ostéocartilagineux médian. Ce manchon osseux est lui-même recouvert par un feutrage conjonctif, riche en lacunes et en capillaires, qu'entoure une mince lamelle ostéo-fibreuse irrégulièrement plissée; l'épithélium branchial recouvre le tout sans interruption.

L'artère et la veine marginale communiquent par un réseau de capillaires disposés en travées longitudinales concentriques qui aboutissent à de petits vaisseaux appliqués contre l'axe ostéo-cartilagineux (pl. XV, fig. 1).

Constitution du kyste parasitaire. - L'examen macroscopique donne, à première vue, l'impression que les parasites sont logés dans une dilatation de la veine marginale; cette impression est inexacte : le point de départ du kyste est toujours à l'extérieur du vaisseau. Les coupes en série permettent de localiser exactement son emplacement. Les parasites se développent toujours dans le tissu conjonctif lacunaire abondamment irrigué qui recouvre la veine marginale. Ce tissu subit de profondes modifications : il est progressivement repoussé vers la périphérie, laminé et partielle- 
ment résorbé, à mesure que les dimensions des parasites augmentent; la mince lamelle ostéo-fibreuse qui l'entoure se déplisse et se renfle en sac, contribuant à former la paroi kystique.

Les modifications les plus intéressantes surviennent du côté de la veine marginale : j'ai observé une résorption totale du manchon osseux périveineux qui devient le siège d'une abondante leucocytose. Ce manchon, épais d'environ $30 \mu$, finit par disparaître entièrement ; la veine marginale, n'étant plus protégée, se déforme par compression mécanique ; sa lumière devient d'abord elliptique, puis ses parois se rapprochent et finissent par entrer en contact : le vaisseau est alors complètement oblitéré (pl. XV, fig. 2).

La localisation constante des kystes à Didymocystis dans le voisinage de la veine marginale (riche en sang artériel) n'est certainement pas fortuite : cet emplacement doit faciliter la nutrition et les oxydations du parasite; il doit favoriser également la dispersion des œufs. Nous ignorons comment se fait cette dissémination, mais il est permis de supposer, avec quelque vraisemblance, que le kyste, arrivé à un certain degré de maturité, s'ouvre spontanément et évacue son contenu à l'extérieur. J'ai recherché, sur les branchies, la présence de cicatrices qui auraient pu correspondre à d'anciens kystes vidés de leur contenu, mais sans succès.

\section{RÉSUMÉ}

En résumé, les lésions provoquées dans la branchie du thon par le développement de Didymocystis wedli, sont les suivantes :

$1^{\circ}$ Hypertrophie très poussée de la région marginale externe du feuillet, région comprise entre la veine et la lamelle périphérique. C'est dans le tissu conjonctif lacunaire et gorgé de sang que se développent les parasites.

$2^{\circ}$ Compression et laminage de ce tissu lacunaire marginal; oblitération complète des capillaires qui le parcourent.

\section{EXPLICATION DE LA PLANCHE XV}

FiG. 1. - Feuillet branchial normal de thon; coupe transversale. Hémalunéosine-orange. $\times 110$.

Fıc. 2. - Fenillet branchial de thon portant un kyste à Didymocystis. Même coloration. $\times 110$. Coupe transversale. La comparaison avec la fig. 1 montre bien la localisation du parasite à l'extérieur de la veine marginale considérablement aplatie et la résorption du manchon osseux périveineux. 

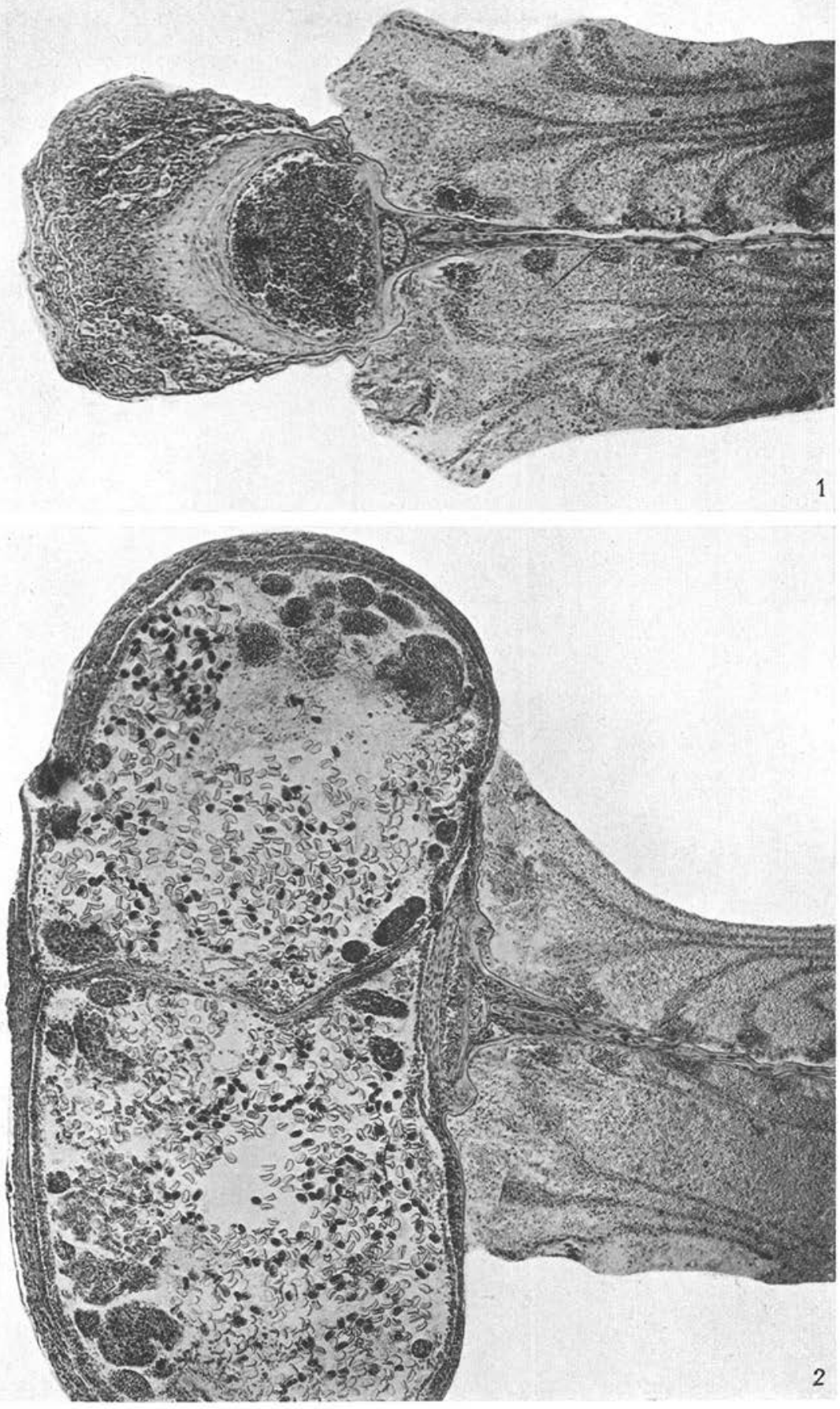

$3^{\circ}$ Résorption phagocytaire du manchon osseux périveineux.

$4^{\circ}$ Aplatissement considérable de la veine marginale, allant jusqu'à l'oblitération complète du vaisseau par accolement de ses parois. La circulation doit être assurée par des suppléances qui s'établissent dans le réseau capillaire voisin.

Telles sont les observations que j'ai été amené à faire sur la structure des kystes à Didymocystis du thon ; elles pourront aider à mieux connaitre la biologie de ces singuliers parasites; il y aura lieu de les compléter par l'étude des formes très jeunes et des premiers stades de Didymocystis chez le thon.

\section{Bibliographie}

Aroola (V.). - Contributo per una monographia dei Didymozoon. I. Didymozoon parassiti del Tono. Arch. Parasit., VI, 1902, p. 99.

BıÉtrix (E.). - Etude de quelques faits relatifs à la morphologie générale du système circulatoire, à propos du réseau branchial des poissons. Thèse Fac. Méd. Paris, 1895.

Dollfus (R:-Ph.). - Sur l'état actuel de la classification des Didymozoonida Monticelli 1888. Ann. Parasit., IV, 1926, p. 149.

FAussek (V.). - Beiträge zur Histologie der Kiemen bei Fischen und Amphibien. Arch. für mikrosk. Anat. u. Entw., LX, 1902, p. 157-174.

Funrmann (O.). — Trematoda in Kükenthal : Handbuch der Zoologie, II, 1928. Kosayashi (H.). - On some digenetic Trematodes in Japan. Parasitology, XII, 1931 , p. $403-404$.

Mac Callum (G. A.) et Mac Callum (W. G.). - The family Koellikeriade (Didymozoonida). Zool. Jahrbüch., Abt. für System, XXXIX, 1916, S. 141-168.

OdHner (T.). - Zur Anatomie der Didymozoen : Ein getrenntgeschlechtlicher Trematode mit rudimentärem Hermaphroditismus. Zoologiska Studier tillägnade Professor T. Tulberg. Uppsala, 1907, p. 309-343.

OKadA (Y. K.). - Description d'un Trématode nouveau : Wedlia katsuzonicola n. sp., seconde espèce du genre. Ann. Parasit., IV, 1926, p. 140-147.

OpPeL (A.). - Lehrbuch der vergleichende Anatomie der Wirbeltiere. Iena, 1905, p. 19-29.

RıEss (J. A.). - Der Bau der Kiemenblätter bei den Knochenfisehen. Arrch. für Naturgesch., XLVII, 1881, I, p. 518-550.

Timon-David (J.). - Sur les Wedlia parasites de l'estomac du thon (Trématodes Didymozoonidæ). Bull. Inst. Océanogr. Monaco, $\mathrm{N}^{\circ} 679,25$ mars 1935.

Wosковоглікоғғ (M. M.). - Der Apparat der Kiemenatmung bei den Fischen. Zool. Jahrb., Abt. f. Anat. u. Ontog. der Tiere, LV, 1932, p. 315,

YAMAGUTi (S.). - Studies on the helminth fauna of Japan. Part. 2, Trematodes of Fishes. Jap. Journ. of Zoology, Tokyo, 31 mars 1934.

(Laboratoire de Zoologie, Faculté des sciences de Marseille). 